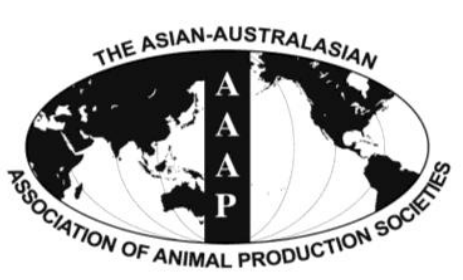

Open Access

Asian Australas. J. Anim. Sci.

Vol. 27, No. 10 : 1499-1512 October 2014

http://dx.doi.org/10.5713/ajas.2014.14060

www.ajas.info

pISSN 1011-2367 elSSN 1976-5517

\title{
An Integrated Epidemiological and Economic Analysis of Vaccination against Highly Pathogenic Porcine Reproductive and Respiratory Syndrome (PRRS) in Thua Thien Hue Province, Vietnam
}

\author{
Haifeng Zhang ${ }^{1}$, Hiroichi Kono*, and Satoko Kubota \\ Department of Animal and Food Hygiene, Obihiro University of Agriculture and Veterinary Medicine, \\ Obihiro, 080-8555, Japan
}

\begin{abstract}
The purposes of this study are to assess pig farmers' preference for highly pathogenic porcine reproductive and respiratory syndrome (PRRS) vaccine, and estimate the cost and benefit of PRRS vaccination in Vietnam. This study employed an integrated epidemiological and economic analysis which combined susceptible-infectious-recovered (SIR) model, choice experiment (CE) and cost-benefit analysis (CBA) together. The result of SIR model showed the basic reproduction number ( $\left.\mathrm{R}_{0}\right)$ of PRRS transmission in this study is 1.3 , consequently, the optimal vaccination percentage is $26 \%$. The results of CE in this study indicate that Vietnam pig farmers are showing a high preference for the PRRS vaccine. However, their mean willingness to pay is lower than the potential cost of PRRS vaccine. It can be considered to be one of the reasons that the PRRS vaccination ratio is still low in Vietnam. The results of CBA specified from the whole society's point of view (Social perspective), the benefits of PRRS vaccination are 2.3 to 4.5 times larger than the costs. To support policy making for increasing the PRRS vaccination proportion, this study indicates two ways to increase the vaccination proportion: i) decrease vaccine price by providing a subsidy, ii) provide compensation of culling only for PRRS vaccinated pigs. (Key Words: Choice Experiment, Cost-Benefit Analysis, Susceptible-infectious-recovered Model, Porcine Reproductive and Respiratory Syndrome, Vietnam)
\end{abstract}

\section{INTRODUCTION}

Highly pathogenic porcine reproductive and respiratory syndrome (PRRS) is a highly contagious, economically devastating disease in pig production. Since 2006, China's pig-farming sector has been damaged by PRRS and has resulted in huge economic losses in the Chinese pig industry (An et al., 2010). This disease quickly spread from China to Vietnam during the Beijing Olympic Games in 2008 (Zhang and Kono, 2012). Total deaths of PRRS infected pigs exceeded 300,000 , and $26 / 60$ provinces were affected during 2008 (Department of Animal Health of Vietnam, 2009).

\footnotetext{
* Corresponding Author: Hiroichi Kono. Tel: +81-155-49-5216, Fax: +81-155-49-5452, E-mail: kono@obihiro.ac.jp

${ }^{1}$ Graduate School of Obihiro University of Agriculture and Veterinary Medicine, Obihiro, 080-8555, Japan.

Submitted Jan. 23, 2014; Revised Apr. 1, 2014; Accepted Apr. 24, 2014
}

Vietnam is a major pork producing country in Asia, producing more than 3 million tons in 2011 (FAOSTAT, 2012). Pig farming is of great importance in Vietnam: $71 \%$ of farm households own pigs (Lemke et al., 2006). Around $80 \%$ of pig production is small-scale, and among those small farms, pig production is the major income source (Lemke et al., 2008). Therefore, PRRS outbreaks severely damage the livelihood of pig farmers (Zhang and Kono, 2012).

For controlling PRRS in Vietnam, three types of control strategies are available ${ }^{1)}:$ i) stamping out (SO), or culling all infected pigs; ii) strategic vaccination (SV), which implies culling all infected pigs and vaccinating susceptible pigs with an optimal vaccination ${ }^{2}$; iii) preventive vaccination, which refers to vaccinating all pigs before the outbreak occurs. The SO control strategy was applied in Vietnam during the outbreak period, and the government provided a subsidy to encourage pig farmers to cull infected pigs. 
Zhang et al. (2013) clarified that the SO strategy is epidemiologically effective and economically beneficial. On the other hand, an epidemiological and economic modeling studied by Zhang et al. (2014) has demonstrated that $\mathrm{SV}$ is more economically efficient control strategy than SO.

The problem of PRRS vaccination in Vietnam is that the vaccination percentage is very low. Only a small part of large commercial pig farms apply PRRS vaccine, which is made in China; and most of the small farms do not accept any PRRS vaccine. On the other hand, the efficacy of "Made in China" vaccine is limited, so the government project on PRRS vaccine development is being carried out in Vietnam ${ }^{3)}$. To increase the PRRS vaccination percentage, information about pig farmers' preference for PRRS vaccination is needed. Furthermore, to evaluate the significance of PRRS vaccination, analysis to estimate the epidemiological effectiveness and economic benefit of PRRS vaccine is also necessary.

Therefore, to support policy making for increasing PRRS vaccination, the purposes of this study are, based on field research to assess pig farmers' preference for PRRS vaccine, and estimate the cost and benefit of PRRS vaccination. It would be hoped that the findings of this research help clarify the most epidemiological effective and economical efficient vaccination strategy for increasing PRRS vaccination in Vietnam.

\section{MATERIALS AND METHOD}

\section{Research area and data}

PRRS outbreaks were severe in the North Central Coast area of Vietnam in 2008 (OIE, 2013). Hue Province is located in the North Central Coast area, and this province has a good reputation for animal disease control in Vietnam (Zhang et al., 2013). Therefore, we selected Hue Province as our study area ${ }^{4)}$. To control PRRS, Hue Province conducted an SO strategy in 2008. During the outbreak period, the pig farmers had to report and cull all pigs of infected herd (Ministry of Agriculture and Rural Development [MARD] regulation No. 80/2008/QD-BNN). On the other hand, the government provided compensation for culling infected pigs. The compensation amounted to about $70 \%$ of the market value of the culled pigs and it encouraged pig farms to report suspicious pigs and cull infected pigs.

In Hue Province, there are eight districts and one large city. Huong Tra District is the main pig farming area, and PRRS severely damaged this district in 2008. The data about pig farming and the PRRS outbreak in Hue Province were collected from four field visits and fieldworks between August 2011 and February 2013. From that fieldwork, epidemiological data were collected from Thua Thien Hue
Department of Animal Health, Vietnam. These epidemiological data included the amount of subsidies provided by local government to PRRS infected farms, dates of subsidy provision, number of infected pigs, bodyweight of culled infected animals and the locations of infected farms. The above data were used in the epidemiological analysis of this study. To obtain detailed information about the PRRS control program, research interviews were conducted with local government officers and experts of agricultural economics at Hue University. We gained valuable detailed information from these interviews for understanding the PRRS outbreak and culling program conducted in this area in 2008.

For data collection of economic analysis, a survey was conducted in villages by staff members of the Hue University of Agriculture and Forestry using an interviewbased questionnaire between February 25th and March 4th, 2013. To clarify the difference in preference for PRRS vaccination between PRRS infected farmers and noninfected farmers, a total of 101 households were surveyed, of which 50 households had been infected by PRRS, and 51 households had not been infected. In order to ensure a balanced selection of households, a set of criteria was developed by the authors and discussed with local veterinarians and an agricultural economic expert of Hue University. These criteria included household involvement or interest in PRRS vaccine. Other criteria were age, years of experience, and size of pig farm.

\section{Choice experiment}

Concerning economic evaluation of animal disease control measures, the cost-benefit analysis (CBA) is a useful tool. However, to determine the cost and benefit of PRRS vaccination, several factors, epidemiological as well as economic, must be taken into account (Morris, 1969). The benefit is the reduction of disease infection by PRRS vaccination. To calculate the benefit, we need to know how many pigs were infected and culled (from epidemiological data), but we also need to know the follow information: i) How many pigs will probably be infected and culled during the vaccination? ii) To control PRRS effectively, how many pigs have to be vaccinated? To address these questions an epidemiological model (susceptible infected recovered [SIR] model) is applied.

The vaccine payment is considered as the cost. However, the PRRS vaccine in this study is a kind of non-marketgoods (the PRRS vaccine considered here is a 'potential' market good that is still under development and not yet marketed). According to expert opinion, the potential cost of this vaccine (including veterinary service) is about 40,000 Vietnam Dong (VND)/pig. This price is reasonable and similar to the price of a China-made vaccine (Field survey, 2013). We use this value as the potential value of 
this vaccine, and compare it with the results of choice experiment (CE). The PRRS outbreak in Vietnam was caused by the newly-emerged virulent strains of the PRRS pathogen virus (HP-PRRS virus). The PRRS vaccine, developed from other strains of the PRRS pathogen virus in other countries might not be effective for controlling the PRRS outbreak in Vietnam. For this reason, the government project on PRRS vaccine development is being carried out in Vietnam (Field survey, 2012).

Therefore, to calculate the cost of the PRRS vaccination program, economic techniques for measuring the value of non-market goods has to be applied. Stated preference approaches have been widely used for this purpose. The CE method, especially has become increasingly popular (Louviere et al., 2000). One of the advantages of the CE approach is its flexibility within applied CBA, and this kind of approach (CE+CBA) has been applied to human healthcare (McIntosh and Ryan, 2002; McIntosh, 2006). However, the studies on CE within CBA in animal disease control are but few in the research literature. Therefore, the CE method was applied to estimate the cost of PRRS vaccination.

The choice experiment studies relating to livestock disease control are relatively few in the research literature. The relatively few articles that report the use of $\mathrm{CE}$ for evaluating people's preferences in relation to livestock disease control include Otieno et al. (2010) who used CE to understand Kenyan farmers' preferences on the type of Disease-Free Zones that would be readily acceptable to them, Bennett and Balcombe (2012) applied CE and contingent valuation to estimate farmers' willingness to pay (WTP) for a Tuberculosis cattle vaccine.

Following the CE design process of Bennett and Balcombe (2012), this research involved a number of stages prior to undertake the survey, designed to ensure that and assumptions used were robust, scientifically realistic and well-grounded in terms of Vietnamese pig farmers' understanding and expectations. These stages involved identification of vaccine attributes, initial questionnaire design, forming a focus group with pig farmers, further development and pretesting of the questionnaire and a pilot survey.

Our survey began with the identification of policyrelevant PRRS control features through an in-depth interview of key officials of the MARD in Hanoi, and local veterinarians in Hue Province. Also, we held focus group discussions with local pig farmers, veterinarians and economic experts of Hue University. Following the guidelines proposed by Bateman et al. (2002), the focus group discussions involved four small-scale pig farmers recruited from Huong Tra District, Hue Province, where PRRS severely occurred in 2008, and two veterinarians from Huong Tra District Veterinary Office, economic experts in Hue University, and the director of the Animal Health Department of Hue province. The focus group discussions were also used to explore important attributes that were identified and their inclusion in the CE. Following pre-testing of the questionnaire, three pilot surveys of four small-scale pig farms were undertaken before the main survey was commenced in order to test the survey method. ${ }^{5)}$

Finally, three attributes were selected for the CE design as follows:

i) Vaccine administration-whether farmers accept or refuse to vaccinate their pigs.

ii) Compensation- subsidy that government pays for farms to cull infected pigs. ${ }^{6)}$

iii) Price of vaccine-vaccine payment per pig per dose.

Attributes of vaccine administration had 2 levels, attribute of price had 3 levels, and attribute of compensation had 4 levels, generated 24 full-profile cards for respondents to fill out (Table 1). Among these, 12 unrealistic profiles were deleted (i.e., the combination of 'Non acceptance of vaccine administration with price of vaccine and compensation'). Respondents were then presented with the full set of 6 pair choices (totaling 12 individual profiles). Prior to answering the survey, respondents were provided an explanation of the hypotheses in the CE question (Table 2). ${ }^{8)}$

The conceptual framework of a CE derives from Lancaster's theory of consumer choice (Lancaster, 1966), which postulates that preferences for goods are a function of the attributes of the goods rather than the goods themselves. An analysis of CE data follows the behavioral framework of random utility theory (McFadden, 1973), which describes discrete choices in a utility maximizing framework. We applied the random parameter logit model (RPL) in the analysis. ${ }^{9)}$

The RPL provides a flexible and computationally practical method for analyzing the results from CE surveys. This model obviates the three limitations of the standard multinomial logit model by the following: i) random taste variation and, hence, explicit accounting for heterogeneity in preferences; ii) unrestricted substitution patterns; and iii) dependence across a panel of repeated choices made by the same respondent, which captures correlation in unobserved factors that affect individual utility (Train, 2003). Furthermore, the RPL is not subject to the strong

Table 1. Attributes and levels for designing choice experiment questions in questionnaire

\begin{tabular}{lc}
\hline Attributes & Levels \\
\hline Vaccine administration & Accept $=1, \quad$ Not accept $=0$ \\
Price of vaccine (VND) & $30,000,40,000,50,000$ \\
Compensation (\%) & $25,50,75,100$ \\
\hline
\end{tabular}

VND, Vietnam Dong. 
Table 2. Hypotheses in choice experiment question

\begin{tabular}{ll}
\hline Hypotheses & \multicolumn{1}{c}{ Explanation } \\
\hline Vaccine efficacy 90\% & $\begin{array}{l}\text { This vaccine was developed in Vietnam. If the pigs were vaccinated, over 90\% of vaccinated pigs can be } \\
\text { prevented from PRRS outbreaks } \\
\text { Pigs can get a PRRS-free certification when administered with the PRRS vaccination. And with this } \\
\text { certification, pigs can be sold at a higher market price. } \\
\text { After administering the vaccination, and if a PRRS outbreak occurs, farmers can get compensation from the } \\
\text { government. But if the vaccination was not administered, then even if an outbreak occurs, the farmers } \\
\text { cannot get any compensation. } \\
\text { Price }\end{array}$ \\
\hline
\end{tabular}

PRRS, porcine reproductive and respiratory syndrome.

assumption of independence of irrelevant alternatives property (Hausman and McFadden, 1984) inherent in the standard multinomial logit model. The specification and estimation of the RPL model follows Revelt and Train (1998), to which the reader is referred for details.

The indirect utility function of the individual $i$ who chooses alternative $j$ in the alternative set $C_{i}$ can be written in the form:

$$
\begin{aligned}
& U_{i j}=V_{i j}+\varepsilon_{i j} \\
& i=1,2,3 \ldots \ldots \ldots n j=1,2,3 \quad \in C_{i}
\end{aligned}
$$

The utility function of this model assumes that the observable component of utility $\mathrm{V}_{\mathrm{ij}}$ is known for each individual $i$ and individual alternative $j$. Without the covariates, with the exception of the error term $\varepsilon_{\mathrm{ij}}$, and without considering the individual attributes, the observable deterministic component of the indirect utility function $V_{i j}$ is:

$$
\mathrm{V}_{\mathrm{ij}}=\beta_{1} \text { Vaccine }_{\mathrm{ij}}+\beta_{2} \text { Compensation }_{\mathrm{ij}}+\beta_{3} \text { Price }_{\mathrm{ij}}
$$

The probability that individual $i$ chooses alternative $j$ is the standard logit formula:

$$
P_{\left(\mathrm{j} \mid \mathrm{V}_{\mathrm{i}}\right)}=\frac{\mathrm{e}^{\mathrm{V}_{\mathrm{ij}}}}{\sum_{q=1}^{j} e^{V_{i q}}}
$$

The main purpose of $\mathrm{CE}$ is to provide information related to how to increase the PRRS vaccination proportion in Vietnam. In addition, based on equation (3), we can estimate the relationship between the 'price of vaccine', 'compensation' and 'percentage' of farms that accept the vaccination. This information is used for cost calculation in CBA.

\section{Modified susceptible infected recovered model}

For calculating the benefit of PRRS vaccination in CBA, an epidemiological model is applied. This kind of analysis method, of an epidemiological model combined with CBA, is well established in previous studies in the field of animal disease management (Bates et al., 2003; Rich and WinterNelson, 2007).

Therefore, we developed a variant of the classic SIR model of PRRS transmission within individual pigs. In the SIR model, we study a population which is composed of three groups of individuals: susceptible (S), infectious (I), and recovered (R). ${ }^{10)}$ A latent state was not included in the model, as the latent period in PRRS infections is thought to be very short, and therefore it has little impact on the infection dynamics (Anderson and May, 1992). This model is based on Lanzas et al. (2008), with modifications specific to PRRS outbreaks in pig farms. This has under the following assumptions:

i) Period of analysis is one year. Only one pig was infected at the beginning of the outbreak. The vaccination was administered immediately just after the first pig was infected.

ii) The pig population mixes homogeneously in terms of piglets, grower pigs, boars and sows. ${ }^{11)}$

iii) All newly introduced (both newborn and from market) animals are susceptible.

iv) All of the infected animals will be identified and culled, under the surveillance system.

v) The population size is constant as the recruitment rate is equal to the exit rate.

vi) The pig population consists of those immune and those susceptible, but they are mixed homogenously and not distinguished in the market.

vii) The infected animals will be identified and culled under the surveillance system $(v I(\mathrm{t}))$, or be sold to the market $(\mu I(\mathrm{t}))$, or will recover and gain natural immunity $(\lambda I(\mathrm{t}))^{12)}$

The dynamics of state transitions are illustrated in Figure 1. The meanings of all symbols are defined in Table 4. This model was defined by a set of three ordinary differential equations. ${ }^{13)}$

$$
\frac{d S_{(t)}}{d_{t}}=-\beta \frac{S_{(t)} I_{(t)}}{N}+(1-\theta)\left(\varphi N+v I_{(t)}\right)+\alpha R_{(t)}-\mu S_{(t)}-\pi S_{(t)}
$$


Table 3. Definitions of variables in SIR model

\begin{tabular}{|c|c|c|c|c|}
\hline Symbol & Unit & Definition & Estimate & Source \\
\hline $\bar{S}$ & Head & Susceptible population & Initial $=28,899$ & Field survey, 2012 \\
\hline$I$ & Head & Infectious population & Initial $=1$ & - \\
\hline$R$ & Head & Recovered population & Initial $=0$ & - \\
\hline$N$ & Head & Total population & Initial $=28.900$ & Field survey, 2012 \\
\hline$N_{v}$ & Head & Total number of vaccinated pigs & Estimated from equation (9) & - \\
\hline$N_{c}$ & Head & Total number of infected and culled pigs & Estimated from equation (10) & - \\
\hline$\beta$ & Day $^{-1}$ & Daily transmission coefficient & 0.21 & $\mathrm{MCMC}^{\mathrm{a}}$ \\
\hline$\varphi$ & Day $^{-1}$ & Daily recruitment rate & 0.0075 & Field survey, $2012^{\mathrm{b}}$ \\
\hline$\theta$ & Day $^{-1}$ & Vaccination proportion & Estimated from equation (8) & - \\
\hline$\alpha$ & Day $^{-1}$ & Resume rate & 0.001 & Field survey, $2012^{\mathrm{c}}$ \\
\hline$\lambda$ & Day $^{-1}$ & Recovery rate & 0.017 & Wills et al., $2002^{\mathrm{d}}$ \\
\hline$v$ & Day $^{-1}$ & Daily culling rate & 0.143 & Field survey, $2012^{\mathrm{e}}$ \\
\hline$\mu$ & Day $^{-1}$ & Daily exit rate & 0.0075 & Field survey, $2012^{\mathrm{f}}$ \\
\hline$h$ & Day $^{-1}$ & Vaccine efficacy & 0.9 & Field survey, $2012^{\mathrm{g}}$ \\
\hline$\pi$ & Day $^{-1}$ & Vaccination proportion reproductive sows & 0.000135 & Roessler et al., $2009^{\mathrm{h}}$ \\
\hline
\end{tabular}

SIR, susceptible infected recovered; PRRS, porcine reproductive and respiratory syndrome.

${ }^{\text {a }}$ The parameter was derived from the epidemiological data, and Markov Chain Monte Carlo was applied (See note 15).

${ }^{\mathrm{b}}$ Daily recruitment rate is the reciprocal number of breeding days (112 days field survey [2012]) times the proportion of the fattening pigs, and plus the reciprocal number of lifetime of sow (3.5 years, Roessler et al. [2009]), times the proportion of the sows in the total population. The total pig population is estimated as 28,900 head, and sow number is estimated as 5,000 head (Field survey, 2012).

${ }^{\mathrm{c}} \alpha$ indicates the probability that PRRS-immunized pigs lose their immunization (expert opinion, $0.1 \%$ of the vaccinated pigs will lose their immunity per day).

${ }^{\mathrm{d}}$ Daily recovery rate is the reciprocal number of infectious periods (60 days, Wills et al. [2002]).

${ }^{\mathrm{e}}$ Daily culling rate is the reciprocal number of the time period between the infected and culled pig (7 days, Field survey [2012]).

${ }^{\mathrm{f}}$ Daily exit rate is same as the daily recruitment rate $(\varphi)$ (also see assumption (4) in SIR model).

${ }^{\mathrm{g}}$ Vaccine efficacy $h$ is the proportion vaccination who obtains immunity; $1-h$ is the proportion of complete failures among the vaccinated. (also see note $8)$.

${ }^{\mathrm{h}} \pi$ is the reciprocal of the lifetime of a sow (=3.5 years), times the proportion of sows in total population $(=5,000 / 28,900)$.

$$
\begin{aligned}
& \frac{d I_{(t)}}{d_{t}}=\beta \frac{S_{(t)} I_{(t)}}{N}-\lambda I_{(t)}-v I_{(t)}-\mu I_{(t)} \\
& \frac{d R_{(t)}}{d_{t}}=\theta\left(\varphi N+v I_{(t)}\right)-\alpha R_{(t)}-\mu R_{(t)}-\lambda I_{(t)}+\pi S_{(t)}
\end{aligned}
$$

The "basic reproduction number $\mathrm{R}_{0}$ " is defined as the average number of successful transmissions per infectious pig (equation 7). And the "Optimal vaccination proportion $\theta$ " is defined as if the vaccination proportion exceeded the optimal vaccination proportion (equation 8 ), then incidence of an infection should decrease (Vynnycky and White,

\begin{tabular}{|c|c|c|c|c|}
\hline \multirow{2}{*}{ Items } & \multicolumn{2}{|c|}{ SO in 2008} & \multicolumn{2}{|c|}{ SV } \\
\hline & Value & Source & Value & Source \\
\hline (1) Vaccine cost (VND/head) & 0 & - & 40,000 & Expert opinion, 2013 \\
\hline (2) Total pig population (head) & 28,900 & Field survey, 2012 & 28,900 & Field survey, 2012 \\
\hline (3) Average body weight of culled pigs (kg) & 50.1 & Field survey, 2012 & 50.1 & Field survey, 2012 \\
\hline (4) Market price of finishing pigs (VND/kg) & 38,000 & MARD $^{1}$ & 38,000 & MARD $^{1}$ \\
\hline (5) Number of culled pigs (heads) & 2,441 & Field survey, $2012^{2}$ & Equation (10) & SIR model \\
\hline (6) Number of vaccinated pigs (heads) & 0 & - & Equation (9) & SIR model \\
\hline (7) Compensation for vaccine (VND/pig) & 0 & - & Equation (3) & $\mathrm{CE}$ \\
\hline $\begin{array}{l}\text { (8) Compensation for culling } \\
\text { ( } \% \text { of market price of cull pigs) }\end{array}$ & 70 & Field survey, $2012^{2}$ & Equation (3) & $\mathrm{CE}$ \\
\hline (9) Loss of PRRS infection (Million VND) & 1,590 & Field survey, $2012^{2}$ & $=(3) \times(4)-(7)) \times(5)$ & SIR model \\
\hline
\end{tabular}

Table 4. Variables used for cost-benefit analysis

SO, stamping out; SV, strategic vaccination; VND, Vietnam Dong; MARD, Ministry of Agriculture and Rural Development; CE, choice experiment; PRRS, porcine reproductive and respiratory syndrome.

${ }^{1}$ MARD set up the market price of pig (38,000 VND/kg) for subsidy calculation (Regulation No.719, June 5th, 2008)

${ }^{2}$ Based on the survey data, in detail refer to Zhang et al. (2013). 
2010). As the number of vaccinated pigs increases, the herd immunity proportion also increases. By decreasing the amount of susceptible people, the disease outbreak subsides. $^{14)}$

$$
\begin{aligned}
& R_{0}=\frac{\beta}{\mu+\lambda+v} \\
& \theta>\frac{1-\left(1 / R_{0}\right)}{h} \quad \text { for } \quad \mathrm{R}_{0}(1-h \theta)<1 \\
& N_{v}=\int_{0}^{t} \theta\left(\varphi N+v I_{(t)}\right) \\
& N_{c}=\int_{0}^{t} v I_{(t)}
\end{aligned}
$$

Based on this modified SIR model, we calculate at least what percentage of pigs $(\theta)$ have to be vaccinated for administering the effective PRRS vaccination (equation 8 , number of pigs that have to be vaccinated [equation 9] and number of pigs infected and culled in the situation with vaccination [equation 10]). Based on those results, we calculate the benefit of the PRRS vaccination.

\section{Costs-benefit analysis}

Given the results of the CE and SIR models, we applied CBA to calculate the costs and benefit of the PRRS vaccination program. Costs-benefit analysis is an economic model for comparing the costs and benefits of the PRRS vaccination program. It has been widely used for evaluating animal disease control programs (Berentsen et al., 1992; Bates et al., 2003). The actual vaccine payment is considered as the cost of the PRRS vaccination. The benefit of the vaccination program is the reduction of disease infection by vaccination (Table 5). The major input variables for economic analysis are summarized in Table 4.

We considered the disease infection and government expenditure use in the outbreak period (2008) as the comparison (control) value for calculating the cost and benefit of the PRRS vaccination. Those values are based on the field survey data. There are two perspectives for calculating the cost and benefit in the CBA, i) private perspective; ii) social perspective (Hitzhusen et al., 1984). The CBA in this study takes both points of view (i.e. private perspective and social perspective). In the social perspective, we consider the whole study area as one unit, therefore, we do not separate the farm's expenditure and government's expenditure for the PRRS vaccination.

According to $\mathrm{CE}$ estimation, there are two ways to increase the vaccination proportion by providing compensation: i) provide compensation to decrease the vaccine price; ii) provide compensation to cull PRRS infected pigs.

The vaccine cost per head per dose is based on expert opinion (Department of Microbiology and Infectious Diseases, Faculty of Veterinary Medicine, Hanoi University of Agriculture, Vietnam). Total pig number, average body weight and the market price of finishing pigs are based on the results of field research. The number of culled pigs and vaccinated pigs were estimated in an epidemiological model (SIR model).

\section{RESULTS AND DISCUSSION}

\section{Choice experiment}

Table 6 gives the estimates of the mean and standard

\begin{tabular}{|c|c|c|c|}
\hline Cost item & Outbreak in 2008 (i) & SV(ii) & Difference (ii-i) \\
\hline \multicolumn{4}{|l|}{ Private perspective } \\
\hline \multicolumn{4}{|c|}{ (1) Government provide compensation for vaccine } \\
\hline Farmer's expenditure (Million VND) & 0 & $(40,000-7) \times(6)$ & (A) Cost of SV \\
\hline Disease loss (Million VND) & 1,590 & (3) $\times(4) \times(5)$ & (B) Benefit of SV \\
\hline Benefit/cost $(\mathrm{B} / \mathrm{C})$ ratio & - & - & $(\mathrm{B}) /(\mathrm{A})$ \\
\hline \multicolumn{4}{|c|}{ (2) Government provide compensation for culling infected pigs } \\
\hline Farmer's expenditure & 0 & $40,000 \times 6$ & (A) Cost of SV \\
\hline Disease loss & 1,590 & (3) $\times(4)-(7) \times(5)$ & (B) Benefit of SV \\
\hline Benefit/cost $(\mathrm{B} / \mathrm{C})$ ratio & - & - & $(\mathrm{B}) /(\mathrm{A})$ \\
\hline \multicolumn{4}{|l|}{ Social perspective } \\
\hline Expenditure & 0 & (1) $\times(6)$ & (A) Cost of SV \\
\hline Disease loss & 4,647 & (3) $\times(4) \times(5)$ & (B) Benefit of SV \\
\hline Benefit/cost $(\mathrm{B} / \mathrm{C})$ ratio & - & - & (B)/(A) \\
\hline
\end{tabular}
deviations of the parameters of the attribute variables. Table 7 shows the results from the RPL model estimation of WTP

Table 5. Costs benefit analysis of PRRS vaccination proportion

PRRS, porcine reproductive and respiratory syndrome; SV, strategic vaccination; VND, Vietnam Dong. The number (1),(2)...(7) ) in Table 5 correspond to the same number in Table 4. 
Table 6. Random parameter logit estimates for PRRS vaccination

\begin{tabular}{lccc}
\hline & \multicolumn{3}{c}{ Coefficient $(t$-ratio $)$} \\
\cline { 2 - 4 } & Non-outbreak & Outbreak & Pooled sample \\
\hline Vaccine & $4.11(2.13)^{* *}$ & $5.09(2.11)^{* * *}$ & $4.56(3.34)^{* * *}$ \\
Compensation & $2.34(10.52)^{* * *}$ & $2.50(16.54)^{* * *}$ & $2.41(18.26)^{* * *}$ \\
Price & $-0.000125(-2.41)^{* *}$ & $-0.000142(-2.87)^{* * *}$ & $-0.000129(-3.75)^{* * *}$ \\
Standard deviation of parameter distributions $(t$-ratio) & & \\
Vaccine & $2.28(2.24)^{* *}$ & $0.47(0.81)$ & $0.43(1.79)^{* *}$ \\
Compensation & $0.64(2.77)^{* * *}$ & $0.81(11.08)^{* * *}$ & $0.79(9.75)^{* * *}$ \\
Log-likelihood & -87.07 & -81.57 & -177.45 \\
McFadden pseudo- $R^{2}$ & 0.74 & 0.75 & 0.74 \\
$\mathrm{n}$ (respondents) & 51 & 50 & 101 \\
$\mathrm{n}$ (choices) & 306 & 300 & 606 \\
\hline
\end{tabular}

PRRS, porcine reproductive and respiratory syndrome.

Statistical significance levels, *** 1\%;** 5\%;*10\%. Corresponding $t$-ratios are shown in parentheses.

for each of the vaccine attributes. Table 8 presents the relationship between the vaccine price, compensation provision and probability of vaccination acceptance.

On the other hand, respondents were asked a number of follow-up questions after the WTP question, to provide some context to their response. The results show that pig farmers believe that PRRS is the most dreadful pig disease, and vaccine alone cannot totally prevent a PRRS outbreak.

The results of CE in this study indicates that Vietnam pig farmers are showing a high preference for the PRRS vaccine $(32,892$ to 35,764 VND in Table 7$)$. However, their mean WTP is lower than the potential cost of PRRS vaccine (40,000 VND/pig). It can be considered to be one of the reasons that the PRRS vaccination ratio is still low in Vietnam. To increase the vaccination ratio, government support for decreasing the vaccine price or providing compensation (for the vaccinated farm only) to cull infected pigs, is a practical solution.

The CE results also show relative high WTP for compensation (of culling infected pigs). Pig farmers think that PRRS is the most dreadful pig disease, and they want to minimize their losses from PRRS, if the outbreak occurs after vaccination. Therefore they set a high value on the compensation that government will pay them if infection occurs even after vaccination.

To diffuse the Vietnam developed PRRS vaccine, PRRS outbreak-experienced farms should be the first target. The result of $\mathrm{CE}$ in this study also indicates that the PRRS outbreak-experienced pig farms are showing a much higher
WTP for the PRRS vaccine. After a long experience of PRRS occurrence in Vietnam, pig farms are eager to administer the potentially highly effective PRRS vaccine. On the other hand, PRRS non-experienced farmers tend to evaluate compensation more. Non-experienced farmers are more likely to avoid the uncertainty of PRRS infection after the vaccination. Therefore, to encourage Vietnam pig farmers to accept the PRRS vaccine, it is important to let them understand more about how effective the vaccine is and what the government support and insurance is for the PRRS vaccination. We suggest that, to diffuse the vaccine, it is better to provide some training seminar with an explanation on the PRRS vaccine, government support for vaccination, and disease knowledge of PRRS.

\section{Modified susceptible infected recovered model}

The basic reproduction number in this study is calculated by equation 7 . The $R_{0}=1.3$, consequently, the optimal vaccination percentage is $26 \%$ (equation 8$)^{16)}$. However, the $R_{0}$ in the study by Charpin et al. (2012) was 2.6 , and accordingly the optimal vaccination percentage should be $68 \%$. (The $R_{0}$ value could vary due to different animal disease management circumstances in different study areas). For sensitivity analysis in the CBA model, we use those two optimal vaccination percentages to calculate the vaccination cost. According to Table 8, to increase the vaccination percentage above $26 \%$ or $68 \%$, two ways can be considered. The first one is for government to provide a subsidy to reduce the vaccine price (reduce the price to

Table 7. Choice experiment estimates of WTP for PRRS vaccination

\begin{tabular}{lccc}
\hline & \multicolumn{3}{c}{ WTP (Standard error, VND) } \\
\cline { 2 - 4 } & Vaccine administration & For 1\% increasing of compensation & Sample size \\
\hline Non-outbreak & $32,892(8,309)^{* * *}$ & $187(68)^{* * *}$ & 51 \\
Outbreak & $35,764(9,759)^{* * *}$ & $176(55)^{* * *}$ & 50 \\
Pooled sample & $35,243(5,101)^{* * *}$ & $187(44)^{* * *}$ & 101 \\
\hline
\end{tabular}

WTP, willingness to pay; PRRS, porcine reproductive and respiratory syndrome; VND, Vietnam Dong.

Statistical significance levels: $* * * 1 \% ; * * 5 \% ; * 10 \%$. Corresponding standard errors are shown in parentheses 
Table 8. Random parameter logit estimates of farmer's preferences for PRRS vaccination

\begin{tabular}{|c|c|c|c|c|c|c|c|}
\hline \multirow{3}{*}{$\frac{\text { Price (VND) }}{70,000}$} & \multicolumn{3}{|c|}{ Compensation $0 \%$} & \multirow{3}{*}{$\begin{array}{c}\text { Compensation (\%) } \\
0\end{array}$} & \multicolumn{3}{|c|}{ Price $=40,000 \mathrm{VND}$} \\
\hline & \multirow{2}{*}{$\begin{array}{c}\text { Probability }(\%)^{1} \\
0.35\end{array}$} & \multicolumn{2}{|c|}{$t$-ratio } & & \multirow{2}{*}{$\begin{array}{c}\text { Probability }(\%) \\
18.66\end{array}$} & \multicolumn{2}{|c|}{$t$-ratio } \\
\hline & & 0.715 & & & & 2.265 & $* *$ \\
\hline 65,000 & 0.72 & 0.810 & & 10 & 22.66 & 2.418 & $* *$ \\
\hline 60,000 & 1.42 & 0.934 & & 20 & 27.22 & 2.609 & $* * *$ \\
\hline 55,000 & 2.80 & 1.103 & & 30 & 32.34 & 2.846 & $* * *$ \\
\hline 50,000 & 5.44 & 1.344 & & 40 & 37.90 & 3.148 & $* * *$ \\
\hline 45,000 & 10.30 & 1.705 & $*$ & 50 & 43.81 & 3.529 & $* * *$ \\
\hline 40,000 & 18.66 & 2.265 & $* *$ & 60 & 49.89 & 4.014 & $* * *$ \\
\hline 35,000 & 31.42 & 3.109 & $* * *$ & 70 & 55.98 & 4.633 & $* * *$ \\
\hline 30,000 & 47.79 & 4.243 & $* * *$ & 80 & 61.89 & 5.424 & $* * *$ \\
\hline 25,000 & 64.65 & 5.705 & $* * *$ & 90 & 67.47 & 6.437 & $* * *$ \\
\hline 20,000 & 78.51 & 7.907 & $* * *$ & 100 & 72.60 & 7.738 & $* * *$ \\
\hline 15,000 & 87.95 & 11.676 & $* * *$ & & & & \\
\hline 10,000 & 93.58 & 18.331 & $* * *$ & & & & \\
\hline 5,000 & 96.68 & 30.156 & $* * *$ & & & & \\
\hline
\end{tabular}

PRRS, porcine reproductive and respiratory syndrome; VND, Vietnam Dong; SIR, susceptible infected recovered.

Statistical significance levels, *** $1 \%$; ** 5\%; * $10 \%$

${ }^{1}$ We selected the values from 'Probability' as the vaccination proportion ' $\theta$ ' in SIR model.

35,000 or 20,000 VND; Table 8 , left part). The second way is for the government to provide compensation to cull infected pigs (provide compensation for $20 \%$ or $100 \%$ of market value; Table 8 , right part). Based on the results of $\mathrm{CE}$ in Table 9, we set the value of the vaccination percentage ' $\theta$ ', and ran the SIR model. ${ }^{17)}$ The total number of vaccinated pigs and infected pigs were estimated by equation 9 and equation 10. Therefore according to the results of the CE and SIR model, four available alternative vaccination programs were designed in two vaccination scenarios (Table 9).

There is an implicated assumption when we apply the results of $\mathrm{CE}$ to the epidemiological and economic analysis framework. That is all the pig farms are the same size. There is further analysis (RPL) which we did not show in the results section, that the larger farm owners have relatively higher WTP. Because of lack of information about farm size in the epidemiological data (of PRRS infection in individual farms), we could not input the factor of 'farm size' into the epidemiological analysis. To diffuse the PRRS vaccination, the larger farms accept the vaccination more easily, but the small farms are a little more difficult. However, even with this limitation, the results of the epidemiological analysis and cost benefit analysis will not change much, because when we ran the SIR model and CBA, we considered all pig farms in the study area as one unit, the number of vaccinated pigs as increasing and the probability of infection decreasing, no matter the farm size. Therefore, further research related to the epidemiological and economic analysis of PRRS vaccination for individual farms is desired.

The epidemiological data were collected from Hue Province where the animal disease surveillance system is well established (Zhang et al., 2013). Our results are under the assumption that all of the infected animals will be identified and culled, under the surveillance system

Table 9. Cost benefit analysis of alternative PRRS vaccination programs

\begin{tabular}{|c|c|c|c|c|c|}
\hline & \multirow{2}{*}{$\begin{array}{c}\text { SO in } 2008 \\
\text { No vaccination }\end{array}$} & \multicolumn{2}{|c|}{ Scenario $1, \theta>26 \%$} & \multicolumn{2}{|c|}{ Scenario $2, \theta>68 \%$} \\
\hline & & Alternative 1 & Alternative 2 & Alternative 3 & Alternative 4 \\
\hline Price of vaccine (VND) & Non vaccine & 40,000 & 40,000 & 40,000 & 40,000 \\
\hline Subsidy for reducing vaccine price (VND/pig) & 0 & 5,000 & 0 & 20,000 & 0 \\
\hline $\begin{array}{l}\text { Compensation for culling infected pigs } \\
(\% \text { of market price of culled pigs })^{1}\end{array}$ & 70 & 0 & 20 & 0 & 100 \\
\hline Vaccination $(\%)$ & 0 & 31.42 & 27.22 & 78.51 & 72.60 \\
\hline Vaccinated number (head) & 0 & $22,613^{2}$ & 19,797 & 49,779 & 46,826 \\
\hline Culled number (head) & 2,441 & 287 & 574 & 24 & 27 \\
\hline
\end{tabular}

PRRS, porcine reproductive and respiratory syndrome; VND, Vietnam Dong; SIR, susceptible infected recovered.

${ }^{1}$ To distinguish the 'government expenditure for reducing the vaccine price' and the 'government expenditure for culling infected pigs', we use the word 'Subsidy' to present the 'government expenditure for reducing the vaccine price', and use the word 'Compensation' to present the 'government expenditure for culling infected pigs'.

${ }^{2}$ Numbers of vaccinated pigs and culled pigs were estimated by modified SIR model. 
(Assumption iv) in SIR model). In other words, an effective surveillance system is essential. The simulation results of this study also show: if the surveillance system is not effective, and the infected pigs are identified and culled more than 3 weeks after the infection, even a $90 \%$ vaccination rate cannot control PRRS outbreaks, and the $\mathrm{B} / \mathrm{C}$ ratio will decrease from 2 to less than $1{ }^{18)}$ Therefore the priority is for implementing economically efficient vaccination strategy in the region where the surveillance system is not well established, in order to enhance the surveillance system.

\section{Costs-benefit analysis}

Based on the results of the CE and SIR models, the costs and benefits of four alternative vaccination programs (Table 9), with private and social perspectives, are presented in Table $10^{19)}$.

From a pig farmer's point of view (private perspective), alternative 3 has the highest $\mathrm{B} / \mathrm{C}$ ratio: 1.55 . It means compared to $\mathrm{SO}$, the benefit of $\mathrm{SV}$ in alternative 3 is 1.5 times higher than the cost of it. However, among the other 3 alternatives, the $\mathrm{B} / \mathrm{C}$ ratios are less than 1 ; it means the cost of SV is higher than the benefit of it. On the other hand, from the whole society's point of view (Social perspective), for all four alternatives, the benefits of SVs are 2.3 to 4.5 times larger than the costs.

Government expenditure for the four alternatives is around 51 to 996 million VND. Compared to government

Table 10. Costs-benefit analysis of SV

\begin{tabular}{|c|c|c|c|c|c|}
\hline & \multicolumn{5}{|c|}{ Private perspective } \\
\hline & SO in 2008 & Alternative 1 & Alternative 2 & Alternative 3 & Alternative 4 \\
\hline \multicolumn{6}{|l|}{ Items } \\
\hline (1) Number of vaccinated pigs & 0 & 22,613 & 19,797 & 49,779 & 46,826 \\
\hline (2) Subsidy $\left(\mathrm{VND} /\right.$ pig) ${ }^{1)}$ & 0 & 5,000 & 0 & 20,000 & 0 \\
\hline $\begin{array}{l}\text { (3) Compensation } \\
\text { (Percentage of market price of culled pigs) }\end{array}$ & 70 & 0 & 20 & 0 & 100 \\
\hline (4) Vaccine cost $(=[40,000-(2)] /$ head $)$ & No vaccine & 35,000 & 40,000 & 20,000 & 40,000 \\
\hline (5) Cost of SV = (1) $\times$ (4) (Million VND) & 0 & 791 & 792 & 996 & 1,873 \\
\hline (6) Number of culled pigs & 2,441 & 287 & 574 & 24 & 27 \\
\hline (7) Average body weight of culled pigs (kg) & 50.1 & 50.1 & 50.1 & 50.1 & 50.1 \\
\hline (8) Market price of finishing pigs (VND/kg) & 38,000 & 38,000 & 38,000 & 38,000 & 38,000 \\
\hline (9) Compensation $=(3) \times 8(\mathrm{VND} / \mathrm{kg})$ & 25,000 & 0 & 7,600 & 0 & 38,000 \\
\hline $\begin{array}{l}\text { (10) Loss from PRRS infection } \\
=6 \times(7) \times(8)-(9) \text { (Million VND) }\end{array}$ & 1,590 & 546 & 874 & 46 & 0 \\
\hline (11) Benefit of SV (Million VND) & - & $1,044^{2}$ & 716 & 1,544 & 1,590 \\
\hline Benefit-cost $(\mathrm{B} / \mathrm{C})$ ratio $=(11 / 5)$ & & 0.69 & 0.90 & 1.55 & 0.85 \\
\hline \multicolumn{6}{|l|}{ Social perspective } \\
\hline (12) Expenditure $=(1) \times 40,000($ Million VND) & 0 & 905 & 792 & 1,991 & 1,873 \\
\hline (13) Cost of SV (Million VND) & 0 & $905^{3)}$ & 792 & 1,991 & 1,873 \\
\hline $\begin{array}{l}\text { (14) Loss from PRRS infection } \\
=(3 \times 4) \times(5) \text { (Million VND) }\end{array}$ & 4,647 & 546 & 1,092 & 46 & 51 \\
\hline (15) Benefit of SV (Million VND) & - & $4,101^{4)}$ & 3,555 & 4.601 & 4.596 \\
\hline Benefit-cost $(\mathrm{B} / \mathrm{C})$ ratio $=(15) /(13)$ & & 4.53 & 4.49 & 2.31 & 2.45 \\
\hline \multicolumn{6}{|l|}{ Total compensation that government provided } \\
\hline Total compensation $($ Million VND) $=(6) \times(7) \times(9)$ & 3,057 & - & 218 & - & 51 \\
\hline Total subsidy $($ Million VND) $=(1) \times(2)$ & - & 113 & - & 996 & - \\
\hline Government budget savings (Million VND) & & $2,944^{5}$ & 2,839 & 2,061 & 3,006 \\
\hline Government budget savings (In percentage) & & $96^{6}$ & 93 & 67 & 98 \\
\hline
\end{tabular}

SV, strategic vaccination; SO, stamping out; VND, Vietnam Dong; PRRS, porcine reproductive and respiratory syndrome.

${ }^{1}$ See note 1) in Table 9.

${ }^{2} 1,044(=1,590-546)$, it is the difference between the disease loss in SO in $2008(1,590)$, and the disease loss in Alternative 1 (546), and so forth.

${ }^{3}$ See note 1) in Table 9.

${ }^{4}$ Similar calculation in note 2$)$.

52,944 (= 3,057-113). It is the difference between the government compensation for SO in 2008 (3,057), and the government compensation for SV in alternative 1 , and so forth.

${ }^{6} 96 \%=(2,944 / 3,057) \times 100 \%$. It is the percentage of 'Government budget saving (Million VND)' over 'Total compensation provision of SO in 2008'. 
expenditure in 2008 in the case of SO (3,057 million VND), 2,061 to 3,006 million VND of government expenditure could be saved if SV was administered instead of SO.

The social benefit of SV is higher than the private benefit. The reason is that in the strategy of SO, the government provided a large amount of compensation (70\% of market price) to cull infected pigs. Therefore, from the farmers' point of view, the loss from disease infection is lower. However, we considered the loss from disease infection within SO as the comparison (control) value in $\mathrm{CBA}$, so the reduction in loss from disease infection (Benefit) by SV is relatively small (i.e. the benefit is calculated by 'loss from disease infection within SO' minus 'the loss from disease infection within SV', because the comparison [control] value [loss from disease infection within SO] is relatively low, so the benefit from the farmers' point of view is also relatively low). On the other hand, from the social perspective, we do not consider government compensation for the benefit calculation, the benefit of SV is much higher than the cost. It means when we consider both government and the pig farmers as a unit, SV has more economic benefit than SO.

The strategic vaccination is also the most budget saving PRRS control strategy. Compared to the outbreak in 2008, if SV was administered instead of SO, the government expenditure could have been reduced from 3,057 million VND to around 51 to 996 million VND (Table 10). Given that the government budget for PRRS control is limited, using government budget efficiently for PRRS control is necessary. If a similar PRRS outbreak were to reoccur now, administration of SV would substantially reduce the budget expenditure compared to SO.

For conducting PRRS vaccination continuously, alternative 3 (provide a subsidy to reduce the price to 20,000 per pig, and increase the vaccination proportion over $68 \%$ ) is the most reasonable. Both farmers and government can benefit from this vaccination alternative. Although from the social perspective, all of the alternatives are beneficial, alternative 3 has more incentives to pig farmers to accept PRRS vaccination. It can be considered that alternative 3 can increase the vaccination proportion more smoothly than other alternatives; and is the most ideal way to diffuse PRRS vaccination in Vietnam.

On the other hand, 'Governmental expenditure for compensation (alternative 2 and alternative 4)' in the results of CBA might be overestimated, since the number of infected and culled pigs shown will mainly occur in the non-vaccinated farms. In that case, as the assumption presented in Table 3 shows, the government does not need to provide compensation to non-vaccinated farms. Therefore, the compensation provision is a more budget saving strategy to diffuse PRRS vaccination. Further research is necessary to collect data related to the government budget for animal disease management, and further analyze of how vaccination affects the government budget and the cost and benefit of PRRS vaccination at the national level is important.

In Vietnam, not only PRRS, but other animal diseases, such as Classical Swine Fever, Food and Month Disease, and Avian Influenza are also occurring now (OIE, 2013). Therefore, the government budget for PRRS control is limited. To diffuse the PRRS vaccine with the limited government budget for PRRS control, SV is more economically beneficial (compared to SO, SV will save $67 \%$ to $98 \%$ of the budget for PRRS control; Table 10 ).

There is a limitation in CBA. PRRS occurs every year in Vietnam, so multiple years' CBA is desired. However, due to the data limitation, only one year epidemiological data was collected, and the analysis period was one year. The multiple years' analysis will be investigated in the next step.

\section{Conclusion remarks}

Compared to other Southeast Asian countries, pig production is extremely important in Vietnam. However, outbreaks of PRRS are still occurring in Vietnam, and SO is still the major control strategy. The Vietnam government still pays a large amount of compensation to cull infected pigs; and there are also severe losses for pig farmers who cull infected pigs. To save the government budget and pig farmer losses from PRRS infection, SV can be considered as a more economically efficient PRRS control strategy. However, the problem of PRRS vaccination in Vietnam is the low rate of vaccination.

To support policy making for increasing the PRRS vaccination proportion, this study indicates two ways to increase the vaccination proportion: i) decrease vaccine price by providing a subsidy, ii) provide compensation for culling infected pigs. The results of CBA show that government support for either decreasing the vaccine price or providing compensation is economically beneficial. On the other hand, the Vietnam-developed vaccine is an assumption in our study. To date, the vaccine used in Vietnam is mainly imported from China, and it is not fully effective. This is considered as one of the reasons that the PRRS vaccination proportion is low in Vietnam. This study gives insight into the possibility of increasing the PRRS vaccination percentage by marketing a Vietnam developed vaccine.

Moreover, this study also shows the high potential benefit of SV (alternative 3). Both pig farmers and the government would benefit if SV was administered. Farmers' income would be increased, and the government costs would be reduced due to the vaccination reducing the 
disease infection substantially. The whole pig production sector also could benefit due to stable pig production (i.e. Vaccination reduces infected and culled pig number, and then total pig production is increased).

\section{Notes}

1) Based on Food and Agriculture Organization (FAO)'s suggestion (Dietze et al., 2011), there are 7 methods that can be considered for PRRS control. To reflect the practical options for PRRS control in Vietnam, and based on suggestion of Rich and Winter-Nelson (2007), this study considers three available PRRS control strategies for analysis.

2) The "Optimal vaccination percentage" is defined as: if this percentage of vaccination is exceeded, then the incidence of an infection should decrease (Vynnycky and White, 2010). Concerning SV, the vaccination must be administered just after the first case of PRRS infection.

3) A few commercial pig farms received administration of the PRRS vaccine experimentally in Vietnam. However, that vaccine was developed from a cultured PRRS virus in China. The PRRS vaccine was made by Guangdong Dahuanong Animal Health Product CO., LTD in China. That is the only government permitted PRRS vaccine available in Vietnam (Field survey, 2013).

4) Southeast Asia has a high pig density, and PRRS outbreaks were mainly in this area. Hue Province has a high pig density and PRRS was severe in this province. Moreover, the small-scale pig production system in Hue is quite similar to other Southeast Asian countries, and according to a local veterinarian's interview, the pig density in Hue is 3 to 5 head per farm, which is similar to the average pig density in Vietnam. Accordingly, some of the results can be generalized to other Asian developing countries. Therefore, we chose Hue as our study area.

5) The first protest survey was conducted in September, 2012. The farmers were confused when answering the original questionnaire, e.g. they believe that vaccinated pigs must have certification, and did not understand why there was an alternative that vaccinated pigs not have certification. We also found it very difficult to explain the CE question if the attributes were more than four. The second pretest survey was conducted in March 2013. From this survey we also made a little modification in CE design. The last pretest survey was conducted just before the main survey began.

6) A subsidy of 25,000 VND per kg of infected pig was paid to PRRS infected farms in 2008. It was about $70 \%$ of the market value of pigs. Based on this information we set the attribute of "Compensation" at four levels: $25 \%, 50 \%$, $75 \%$, and $100 \%$.

7) The PRRS vaccine which is made in China is 39,000 VND per dose, and one dose is sufficient (Field survey,
2012). Base on this information, we set the attribute of "Price of vaccine" at three levels: 30,000, 40,000, and 50,000 (VND per dose per pig).

8) Although the vaccine efficacy was announced as $95 \%$ (Zhuyiwang, 2009), to consider the different pig farming situations between China and Vietnam, we conservatively postulated the vaccine efficacy (of a Vietnam-developed vaccine) $h$ as $90 \%$.

9) Either the RPL or latent class model could be used to investigate preference heterogeneity. There are no theoretical grounds for the choice of one over the other (Green and Hensher, 2003). We explored both approaches, but found the RPL to fit the sample data better.

10) In regard to " $R$ ", originally, it represents the individuals who have an immune response by naturally "recovered" (Kermack and McKendrick, 1927). On the other hand, the SIR model in this study is a modified version, and this category " $R$ " represents the pigs that have an "immune" response by both naturally "recovered" and "vaccinated". To emphasize this point, we used "immune" instead of "recovered" to describe the category "R".

11) According to the local veterinarian's estimation, the total number of pigs in the Huong Tra District in 2008 was around 28,900. It was impossible to classify the pigs in this area into categories, for example, piglets, fattening pigs, sows, and so we assumed the distribution of pigs to be homogeneous with an average body weight of $50.1 \mathrm{~kg}$. Moreover, feed cost can be considered to differ in various situations according to the different control strategies; however, because detailed data regarding pig feeding are also not available, we did not consider the feed cost in this study, and focused only on the disease control cost. In Vietnam, both the PRRS vaccinated and the non-vaccinated pigs are sold, but not distinguished in the market.

12) The detection ability of the surveillance system as applied in practice is likely to be less than $100 \%$. It is also a rational assumption that some of immunized pigs might lose their immunity during the outbreak $\left(\alpha R_{(t)}\right)$. According to expert opinion, $0.1 \%$ of the immunized pigs might be expected to lose their immunity per day $(\alpha=0.001)$.

13) We considered the frequency-dependent (true mass action) transmission in our SIR model. The HP-PRRS transmission rate $\left(\beta \frac{S(t) I(t)}{N}\right)$ will depend on the pig population size. Therefore, following the methods of De Jong (1995), assuming true mass action, the transmission rate will be $\left(\beta \frac{S(t) I(t)}{N}\right)$ in our SIR model.

14) $R_{0}$ is formally defined as the average number of secondary infectious pigs resulting from a typical infectious pig following its introduction to a totally susceptible population. For the purpose of predicting the threshold level of vaccination necessary for eradication, and based on 


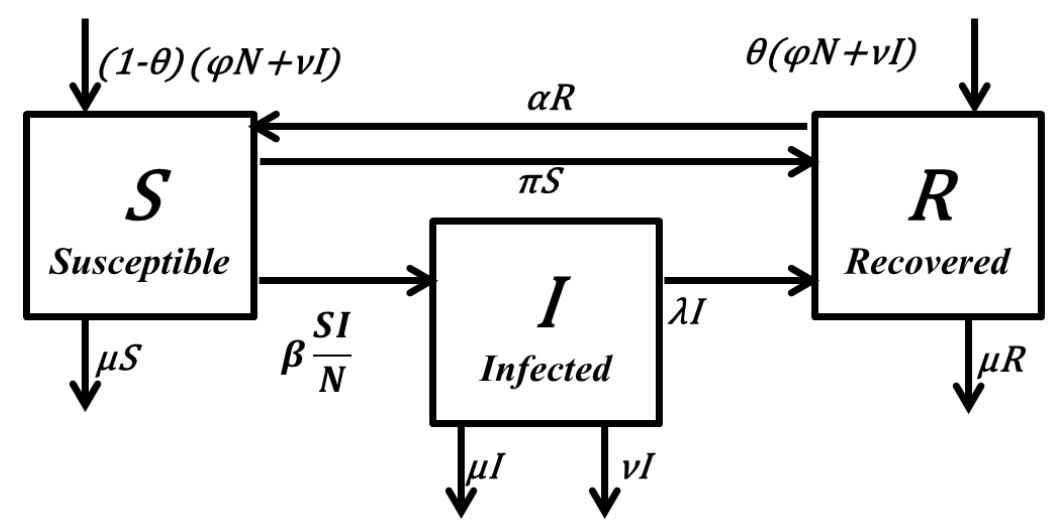

Figure 1. Flow diagram of the SIR model for PRRS control. SIR, susceptible infected recovered; PRRS, porcine reproductive and respiratory syndrome.

Keeling and Rohani (2008) (pp. 26-28), the calculation of $R_{0}$ is described in equation (4).

Vaccination increases the proportion of immunized pigs and decreases the proportion of susceptible pigs, resulting in fewer transmissions from each infected pig. In such circumstances, with the proportion of susceptible pigs decreasing, the number of actual disease transmissions will fall to less than $R_{0}$, and is defined as the net or effective reproduction number, often given the symbol $R_{\mathrm{n}}$. In simplest terms,

$$
R_{n}=R_{0} \times \mathrm{S}
$$

where $s$ is defined as the proportion of the population that is susceptible. Equation (a) shows a fundamental relationship that when the proportion susceptible, $s$, is equal to $1 / R_{02}$ then each infectious person should lead to just a single transmission, i.e., $R_{0}=1$. If the proportion susceptible is less than this proportion, incidence will decrease; if it is greater, incidence will increase. This critical threshold of susceptibility is typically described in terms of its converse, or the proportion immune $(=1-s)$. Therefore, the critical threshold of immunity, defined as the optimal vaccination proportion, is then given by equation (5). A detailed explanation is presented in Vynnycky and White (2010).

15) Epidemiological data was collected from the field survey. Parameter $\beta$ was derived from those data by applying Markov Chain Monte Carlo (MCMC) methodology. MCMC with 2,000 iterations of Gibbs sampling was performed using an EXCEL spreadsheet. Details of MCMC are refered in Wakui (2009) and Nodelijk et al. (2000).

16) From the field survey, 2,441 head were culled; on the other hand, according to the SIR model, based on the stamping out strategy, the total culled heads were estimated at 2,882 . The simulated culled number was very close to the exact number, therefore we consider this epidemiological model to be appropriate.

17) As indicated in Figure 1, our SIR model considered that time is required to administer the vaccination, in line with the actual situation in developing countries, and it also considered the time required for spread of the virus (Zhang et al., 2013). However, this model still differs from the real situation, in which there is a time lag between vaccine administration and acquirement of immunity. This time lag varies according to pig breed, age, and PRRS pathogen strain. Further research is required to evaluate this time lag in the SIR model.

18) The infectious period is the time period between the pig being infected and culled. We assumed the surveillance was adequately functional and the infectious period was 1 week (parameter $v=0.143$ ) in the epidemiological simulation. However, if the surveillance system is not functionally efficient, and the infectious period increases to more than 3 weeks (parameter $v=0.048$ ), the outbreak will continue for more than 1 year, which is outside our period of analysis.

19) According to the local veterinarian's estimate, the total number of pigs in Huong Tra District in 2008 was around 28,900 . It was impossible to classify the pigs in this area into several categories, for example, piglet, fattening pigs and sows, so we assumed the distribution of pigs to be homogeneous with an average body weight of $50.1 \mathrm{~kg}$. Moreover, we considered that the feed cost was different in different situations under different control strategies; however, because detail data of pig feeding were also not available, we didn't consider the feed costs in this study, and only focused on the disease control cost.

\section{ACKNOWLEDGMENTS}

This work was supported by the earmarked fund for Modern Agro-industry Technology Research System. 


\section{REFERENCES}

An, T., Z. Tian, Y. Xiao, R. Li, J. Peng, T. Wei, Y. Zhang, Y. Zhou, and G. Tong. 2010. Origin of highly pathogenic porcine reproductive and respiratory syndrome virus, China. Emerg. Infect. Dis. 16:365-367.

Anderson, R. and R. May. 1992. Infectious Diseases of Humans: Dynamics and Control. Oxford University Press. Oxford, UK.

Bateman, I. J., R. T. Carson, B. Day, M. Hanemann, N. Hanley, T. Hett, M. Jones-Lee, G. Loomes, S. Mourato, E. Ozdemiroglu, D. W. Pearce, R. Sugden, and J. Swanson. 2002. Economic Valuation with Stated Preference Techniques: A Manual. Edward Elgar, Cheltenham, UK.

Bates, T. W., T. E. Carpenter, and M. C. Thurmond. 2003. Benefitcost analysis of vaccination and preemptive slaughter as a means of eradicating foot-and mouth disease. Am. J. Vet. Res. 64:805-812.

Bennett, R. and K. Balcombe. 2012. Farmers' willingness to pay for a tuberculosis cattle vaccine. J. Agric. Econ. 63:408-424.

Berentsen, P., A. Dijkhuizen, and A. Oskam. 1992. A dynamic model for cost-benefit analyses of foot-and-mouth disease control strategies. Prev. Vet. Med. 12:229-243.

Charpin, C., S. Mahe, A. Keranflech, C. Belloc, R. Cariolet, M. F. Potier, and N. Rose. 2012. Infectiousness of pigs infected by the Porcine Reproductive and Respiratory Syndrome virus (PRRSV) is time-dependent. Vet. Res. 2012:43-69.

De Jong, M. C. M. 1995. Mathematical modeling in veterinary epidemiology: Why model building is important. Prev. Vet. Med. 25:183-193.

Dietze, K., J. Pinto, S. Wainwright, and C. Hamilton. 2011. Porcine reproductive and respiratory syndrome (PRRS): virulence jumps and persistent circulation in Southeast Asia. In Focus on..., 5, p. 8. Food and Agriculture Organization of the United Nations.

Greene, W. H. and D. A. Hensher. 2003. A latent class model for discrete choice analysis: Contrasts with mixed logit. Transp. Res.Part B. Methodol. 37:681-698.

Hausman, J. and D. McFadden. 1984. Specification tests for the multinomial logit model. Econometrica. 52:1219-1240.

Holtkamp, D., K. James, Z. Jeff, N. Eric, R. Hans, Y. Tiffany, W. Chong, Y. Paul, and H. Charles, 2012. Economic analysis of PRRS virus elimination from a herd. Iowa state university animal industry 2012. http://www.ans.iastate.edu/report/air/2012pdf/R2678.pdf Accessed January 23, 2014.

Hitzhusen, F., B. Macgregor, and D. Southate. 1984. Private and social cost-benefit perspectives and a case application on reservoir sedimentation management. Water Int. 9:181-184.

Keeling, M. and P. Rohani. 2008. Modeling infectious disease in humans and animals. Princeton University Press, Princeton, NJ, USA.

Kermack, W. O. and A. G. McKendrick. 1927. A contribution to the mathematical theory of epidemics. Proc. R. Soc. (London) 115:700-721.

Lancaster, K. 1966. A new approach to consumer theory. J. Polit. Econ. 74:132-157.

Lanzas, C., S. Brien, R. Ivanek, Y. Lo, P. P. Chapagain, K. A. Ray, P. Ayscue, L. D. Warnick, and Y. T. Grohn. 2008. The effect of heterogeneous infectious period and contagiousness on the dynamics of Salmonella transmission in dairy cattle. Epidemiol. Infect. 136:1496-1510.

Lemke, U., B. Kaufmann, L. T. Thuy, K. Emrich, and A. Valle Zárate. 2006. Evaluation of smallholder pig production systems in North Vietnam: Pig production management and pig performances. Livest. Sci. 105:229-243.

Lemke, U., M. Mergenthaler, R. Rössler, L. Huyen, P. Herold, B. Kaufmann, and A. Valle-Zárate. 2008. Pig production in Vietnam - A review. CAB Reviews: Perspectives in Agriculture, Veterinary Science, Nutrition and Natural Resources. 23:1-15.

Louviere, J., D. A. Hensher, and J. Swait. 2000. Stated Choice Methods: Analysis and Application. Cambridge University Press, Cambridge, UK.

McFadden, D. 1973. Conditional logit analysis of qualitative choice behavior. In: Frontiers in Econometrics (Ed. P. Zarembke). Academic Press, New York, USA. pp. 447-470.

McIntosh, E. 2006. Using discrete choice experiments within a cost-benefit analysis framework. Pharmacoeconomics 24:855868.

McIntoch, E. and M. Ryan. 2002. Using discrete choice experiments to derive welfare estimates for the provision of elective surgery: Implications of discontinuous preferences. J. Econ. Psychol 23:367-382.

Morris R. 1969. Assessing the economic value of veterinary services to primary industries. Aust. Vet. J. 45:295-300.

Nodelijk, G., M. C. M. D. Jong, A. V. Nes, J. C. M. Vernooy, L. A. M. G. V. Leengoed, J. M. A. Pol, and J. H. M. Verheijden. 2000 Introduction, persistence and fade-out of porcine reproductive and respiratory syndrome virus in a Dutch breeding herd: a mathematical analysis. Epidemiol. Infect. 124:173-182.

Otieno, D. J., E. Ruto, and L. Hubbard. 2011. Cattle farmers' preferences for disease-free zones in Kenya: An application of the choice experiment method. J. Agric. Econ. 62:207-224.

Revelt, D. and K. Train. 1998. Mixed logit with repeated choices: Household's choices of appliance efficiency level. Rev. Econ. Stat. 80:647-657.

Rich, K. M. and A. Winter-Nelson. 2007. An integrated epidemiological-economic analysis of foot and mouth disease: Applications to the southern cone of South America. Am. J. Agric. Econ. 89:682-697.

Roessler, R., P. Herold, A. Willan, H. P. Piepho, L. T. Thuy, and A. Valle Zarate. 2009. Modelling of a recording scheme for market-oriented smallholder pig producers in Northwest Vietnam. Livest. Sci. 123:241-248.

Train, K. E. 2003. Discrete Choice Methods with Simulation. Cambridge University Press, New York, NY, USA.

Vynnycky, E. and R. White. 2010. An Introduction to Infectious Disease Modeling. Oxford University Press, New York, NY, USA.

Wakui, Y. 2009. Dougu tosite no beizutoukei (Analyzing tool of Bayesian statistics). Nippon Jitugyou Publishing, Tokyo, Japan. (in Japanese).

Wills, R. W., A. R. Doster, and F. A. Osorio. 2002. Transmission of porcine reproductive and respiratory syndrome virus (PRRSV) to age-matched sentinel pigs. J. Swine Health Prod. 10:161165. 
Zhang, H. and H. Kono. 2012. Economic impacts of porcine respective and respiratory syndrome (PRRS) outbreak in Vietnam pig production. Trop. Agric. Res. 23:152-159.

Zhang, H., H. Kono, S. Kubota, N. T. M. Hoa, and N. V. Hung. 2013. Epidemiologic and economic analysis of porcine HPPRRS outbreak control in Vietnam. J. Agric. Dev. Stud. 23:3142.
Zhang, H., H. Kono, H. Makita, and S. Kubota. 2014. An integrated epidemiological-economic analysis of HP-PRRS control strategies in Vietnam, Japanese J. Rural Econ. 16:57-64. Zhuyiwang (Pig news). 2012. New attenuated PRRS vaccine is on sale. 27th May, 2009, (In Chinese). http://attenuatedcj.zhue.com.cn/jinrijujiao/qiyexinwen/shouyao /200905/27-72566.html Accessed November 21, 2012. 